NBI-HE-92-20

CERN-TH.6508/92

\title{
Parity breaking at high temperature and density
}

\author{
J. Ambjørn and K. Farakos凹 \\ The Niels Bohr Institute \\ Blegdamsvej 17, DK-2100 Copenhagen Ø, Denmark \\ and \\ M. E. Shaposhnikov \\ CERN, CH-1211 Geneva 23, Switzerland
}

\begin{abstract}
We investigate the question of parity breaking in three-dimensional Euclidean $S U(2)$ gauge-Higgs theory by Monte Carlo simulations. We observe no sign of spontaneous parity breaking in the behaviour of both local and non-local gauge invariant operators. However, the presence of parity odd terms in the action can induce a phase transition to a parity odd ground state which is characterized by a Chern-Simons like condensate. The implications for various proposed scenarios of fermion number non-conservation is discussed.
\end{abstract}

\footnotetext{
${ }^{1}$ Supported by an E.E.C Fellowship

Permanent address: National Technical University of Athens, Depart. of Physics,GR 15773 Athens, Greece.

${ }^{2}$ On leave of absence from the Institute for Nuclear Research of the Russian Academy of Sciences, Moscow 117312, Russia
} 


\section{Introduction}

The non-conservation of the fermion number in the electroweak theory [1] due to the anomaly in the fermionic current has, in recent years, attracted a lot of attention. Under ordinary conditions the processes associated with baryon number non-conservation are exponentially suppressed, since they correspond to tunnelling between different classical vacua connected by topological non-trivial gauge transformations. However, it has recently been realized that there can be a great amplification of anomalous fermion-number non-conservation. Generally, this might occur when the energy stored in the system is big enough. In principle, the energy can be of different forms. The simplest example is provided by a system at high temperatures [2] and/or large fermionic densities [3]. Otherwise, we can consider decays of superheavy fermions [4] or collisions of particles at high energies [5, 6].

It has been suggested recently to combine non-conservation of the baryon number with the possibility of spontaneous parity breaking $[7,8,9,10]$ in the high temperature limit [11] of the electroweak theory and in this way explain the observed baryon asymmetry of the universe entirely within the context of the electroweak theory [12]. The scenario is as follows. At temperatures higher than $T_{c}$, corresponding to the electroweak transition temperature $T_{c}(\sim O(100) \mathrm{GeV})$ the $S U(2)$ symmetry is restored, but (by assumption) parity is spontaneously broken. Since parity is a discrete symmetry this leads to the creation of domains with different signs of parity breaking inside them. However, due to a small but explicit breaking of $C P$ coming from the KM-matrix, one type of domain will be energetically favourable, and will eat the domains with opposite parity. When the universe has cooled to $T \simeq T_{c}$ it will be in a state of maximal parity asymmetry, and the expectation values of parity odd operators, which are naturally present in the high temperature phase, will be different from zero. Of particular interest in this connection is the Chern-Simons condensate, which appears as a term in the effective three-dimensional high temperature action. After the electroweak phase transition we have a situation where the gauge symmetry is broken, but the parity invariance is restored. The Chern-Simons condensate, characterized by a non-zero expectation value of the Chern-Simons den-

sity $n_{c s}$, will disappear when parity invariance is restored. Due to the anomaly of the fermion number current, the expectation value $\left\langle n_{c s}>\right.$ in the parity broken phase may be converted to fermions, thereby explaining the baryon asymmetry observed in the universe. One additional assumption necessary for the above scenario to be viable is that the electroweak transition at $T_{c}$ is first order [2, 12]. If it is second order, the generated baryon asymmetry will be washed away after the transition, by the very same baryon number violating processes. The reason is that the effective, temperature-dependent masses changes smoothly from zero after a second- 
order transition. Consequently, the barrier separation of the different classical vacua will grow slowly and there will be sufficient time to create a new thermal equilibrium between baryons and anti-baryons.

Obviously a large number of assumptions go into the above suggested mechanism for generation of the observed baryon asymmetry, and it would be preferable if one could check some of them. A determination of the order of the electroweak transition seems difficult, both from an analytic point of view [13, 14], or by means of lattice gauge simulations[15]. The assumption that parity is spontaneously broken at high temperature is more accessible to numerical investigation, since the effective high temperature limit of the static magnetic sector of the electroweak theory is described by the three-dimensional $S U(2)$ gauge-Higgs system [16, 17]. In the broken phase of the three-dimensional gauge-Higgs theory it seems impossible to have spontaneously broken parity [1], but in the unbroken phase, which is the one of interest in the above cosmological context, infra-red singularities make a perturbative analysis unreliable. It is the purpose of the present paper to perform a non-perturbative analysis of the problem of parity breaking using the technique of lattice gauge theories.

Due to the explicite breaking of parity and CP-invariance, the effective action for the gauge and Higgs fields contains a number of parity odd terms. The ChernSimons term has already been mentioned. It is given by

$$
n_{c s}=\frac{1}{16 \pi^{2}} \varepsilon_{i j k} \operatorname{Tr}\left(F_{i j} A_{k}-\frac{2}{3} A_{i} A_{j} A_{k}\right)
$$

It appears, for example, in the presence of the non-zero fermionic number density [18]. The existence of a Higgs field leads to other parity odd operators when the fermions are integrated out. The simplest operator is

$$
\tilde{\mathcal{O}}_{1}^{\text {cont }}=i \varepsilon_{i j k} F_{i j}^{a} \varphi^{\dagger} \tau^{a} \stackrel{\leftrightarrow}{D}_{k} \varphi
$$

The triangle diagram leading to this operator is shown in fig.1 and involves the coupling of two Higgs fields to an $S U(2)$ gauge field. Clearly, there is an infinite set of such operators, corresponding to various one-loop diagrams. A simple example is

$$
\tilde{\mathcal{O}}_{2}^{c o n t}=i \varepsilon_{i j k} \varepsilon^{a b c}\left(\varphi^{\dagger} \tau^{a} \stackrel{\leftrightarrow}{D}_{k} \varphi\right)\left(\varphi^{\dagger} \tau^{b} \stackrel{\leftrightarrow}{D}_{k} \varphi\right)\left(\varphi^{\dagger} \tau^{c} \stackrel{\leftrightarrow}{D}_{k} \varphi\right)
$$

The pentagon diagram which leads to $\tilde{\mathcal{O}}_{2}^{\text {cont }}$ in the high temperature limit is also shown in fig 1.

The coefficients in front of the terms (11)-(3) will be extremely small. They have their origin in the CP-breaking part of the electroweak theory. Although small, they might still be important if parity is spontaneously broken, as discussed in 
[11. In the first part of this article we will mainly consider the terms as small perturbations to the underlying three-dimensional gauge-Higgs system, since the main purpose is to find traces of spontaneous parity breaking and one way to do that is by adding parity-odd operators and measuring the response. From this point of view the operators $\tilde{\mathcal{O}}_{1}^{\text {cont }}$ and $\tilde{\mathcal{O}}_{2}^{\text {cont }}$ are in many respects more convenient than the Chern-Simons operator (11). They are, contrary to (11), invariant under local gauge transformation. This means that they have a natural implementation on the lattice which preserves the parity odd nature of the terms. There exists no simple and natural lattice implementation of the Chern-Simons density (1). Nevertheless (2) and (3) have the same origin in the context of an effective high temperature expansion of the electroweak theory, and they are related to the Chern-Simons term (1) since their sum for a constant Higgs field $\varphi_{0}$ is equal to $n_{c s}$ :

$$
\tilde{\mathcal{O}}_{1}^{\text {cont }}+\tilde{\mathcal{O}}_{2}^{\text {cont }}=\left|\varphi_{0}\right|^{2} n_{c s}
$$

The use of terms like (2) and (3) might therefore be superior to earlier attempts 22 to add directly the Chern-Simons term as a small perturbation to the effective high temperature lattice action and in this way test the properties of the vacuum of the three-dimensional theory. The outcome of these earlier attempts wos ambiguous, and the ambiguity seems to be related to our inability to find a physical acceptable representation of the Chern-Simons density on the lattice.

Since there is an infinite number of terms like (2) and (3), we will in this article confine ourselves to the study of theories where only the simplest source term (2) is added. We add it in two different versions, namely as given by (2) and in a "non-local" version:

$$
\tilde{\mathcal{O}}_{3}^{\text {cont }}=\tilde{\mathcal{O}}_{1}^{\text {cont }} /\left|\varphi^{2}\right|
$$

The reason for considering $\tilde{\mathcal{O}}_{3}^{\text {cont }}$ can be found in eq. (幽. In principle we are interested in adding the Chern-Simons term as a source term, but as mentioned above we are unable to do so directly, and the non-polynomial interaction (5) might be a good approximation. In the phase where the gauge symmetry is spontaneusly broken and $\varphi$ has only small fluctuations around a vacuum expectation $\left\langle\varphi_{0}\right\rangle \neq 0$ there should not be any differencef between the source terms (2) and (5). There could be significant differences in the unbroken phase where $\left\langle\varphi_{0}\right\rangle=0$, and it is our hope that the term (5) would capture a possible difference in this phase between adding a source term like (11) and a source term like (2).

Since the lattice Monte Carlo simulations always use a finite volume, we cannot strictly speaking have a genuine transition to a parity odd phase. If spontaneous

\footnotetext{
${ }^{3}$ We use here the standard continuum notion of spontaneous symmetry breaking. In the lattice simulation the gauge is not fixed and $\langle\varphi\rangle=0$.
} 
symmetry breaking is a possibility for the system at infinite volume, one would nevertheless observe a clear signal by adding the operator $\tilde{\mathcal{O}}_{1}^{\text {cont }}$ as a source term, since the expectation value of $\tilde{\mathcal{O}}_{1}^{\text {cont }}$ should grow with the volume of the system. We have illustrated the situation of spontaneous symmetry breaking as compared to no symmetry breaking in fig.2. In the case of no spontaneous symmetry one would expect to observe a linear growth of $\left\langle\tilde{\mathcal{O}}_{1}^{\text {cont }}\right\rangle$ as a function of the coupling strength $\mu$, and almost no volume dependence should be present. In the following we will report on the results of $\mathrm{MC}$ simulations trying to distinguish between the two situations of fig. 2.

\section{The phase diagram with $\tilde{\mathcal{O}}_{1}^{\text {cont }}$ in the action}

For the three-dimensional gauge Higgs system we use the standard lattice action which is given by:

$$
\begin{aligned}
S= & \frac{\beta_{G}}{2} \sum_{x ; \mu \nu} \operatorname{Tr}\left(1-U_{\square(x) ; \mu \nu}\right)+\beta_{R} \sum_{x}\left(\frac{1}{2} \operatorname{Tr} \Phi_{x}^{\dagger} \Phi-v^{2}\right)^{2}+ \\
& \frac{\beta_{H}}{2} \sum_{x ; \mu} \operatorname{Tr}\left(\Phi_{x}-U_{x, x+\mu} \Phi_{x+\mu}\right)^{\dagger}\left(\Phi_{x}-U_{x, x+\mu} \Phi_{x+\mu}\right) .
\end{aligned}
$$

Here $U_{x, x+\mu} \in S U(2)$ denotes the gauge variable associated with the link $(x, x+\mu)$, $U_{\square(x), \mu \nu}=U_{x, x+\mu} U_{x+\mu, x+\mu+\nu} U_{x+\mu+\nu, x+\nu} U_{x+\nu, x}$ the variable associated with the lattice point $x$ and the plaquette $\square$ spanned by the unit lattice vectors $\hat{\mu}, \hat{\nu}$. Finally $\Phi_{x}$ denotes the $S U(2)$ Higgs doublet field associated lattice point $x$, but written in matrix form:

$$
\Phi \equiv\left(\begin{array}{cc}
\phi_{1} & -\phi_{2}^{*} \\
\phi_{2} & \phi_{1}^{*}
\end{array}\right)=R \cdot V, \quad R \in \mathbf{R}_{+}, V \in S U(2)
$$

By taking the vacuum expectation value $v^{2}$ as the following function of the coupling constants:

$$
v^{2}=\frac{2 \beta_{R}+3 \beta_{H}-1}{2 \beta_{R}}
$$

the naive continuum limit of the theory is obtained by scaling $\beta_{R} \rightarrow 0$ as $a$ while $\beta_{H} \rightarrow 1 / 3$ such that $v^{2} \rightarrow 0$ as $a$, and (6) goes to the following continuum action:

$$
\mathcal{L}=\frac{1}{4 g^{2}} F_{\mu \nu}^{2}+\left|D_{\mu} \phi\right|^{2}+M_{c}^{2}|\phi|^{2}+\lambda_{c}|\phi|^{4}
$$

The tree value connection between the lattice parameters in (6) and the continuum coupling constants in (9) is as follows

$$
M_{c}^{2}=\frac{2\left(1-2 \beta_{R}-3 \beta_{H}\right)}{\beta_{H} a^{2}}
$$




$$
\begin{aligned}
\lambda_{c} & =\frac{4 \beta_{R}}{\beta_{H}^{2} a} \\
g_{c}^{2} & =\frac{4}{\beta_{G} a}
\end{aligned}
$$

We now add the parity odd source term to the action (6). First we define lattice quantities corresponding to $F_{\mu \nu}^{a}(x)$ and $\varphi^{\dagger} \tau^{a} \stackrel{\leftrightarrow}{D}_{\mu} \varphi$ :

$$
\begin{gathered}
\frac{i}{2} F_{\mu \nu}^{a}(x) \rightarrow G_{\mu \nu}^{a}(x)=\frac{1}{8} \operatorname{Tr} \tau^{a}\left[U_{\square(x) ; \mu, \nu}+U_{\square(x) ; \nu,-\mu}+U_{\square(x) ;-\mu,-\nu}+U_{\square(x) ;-\nu, \mu}\right] \\
\frac{1}{2} \varphi^{\dagger} \tau^{a} \stackrel{\leftrightarrow}{D}_{\mu} \varphi \rightarrow H_{\mu}^{a}(x)=\frac{1}{4} \operatorname{Tr} \Phi_{x}^{\dagger} \tau^{a}\left[U_{x, x+\mu} \Phi_{x+\mu}-U_{x, x-\mu} \Phi_{x-\mu}\right]
\end{gathered}
$$

and the lattice action can then be written as

$$
S_{1}=4 \mu \sum_{x} \varepsilon_{\mu \nu \lambda} H_{\mu}^{a}(x) G_{\nu \lambda}^{a}(x)
$$

These lattice terms are chosen in such a way that they have the correct symmetry, even at the discrete level, and such that the naive continuum limit of the rhs of (11) and (12) agree with the lhs. The naive continuum limit of the new term is obtained by scaling $\mu \rightarrow \mu_{c} / a$ while keeping the above-mentioned standard scaling of the other couplings. The fact that the continuum coupling constant in front of a term like $\tilde{\mathcal{O}}_{1}^{\text {cont }}$ has the dimension of length reflects that $\tilde{\mathcal{O}}_{1}^{\text {cont }}$ is not a renormalizable term.

Let us first discuss the phase diagram in the case where the source term $\mu S_{1}$ is zero. According to our knowledge no detailed investigation has been made for the model given by (6) in three dimensions. A careful study of a three-dimensional model where the Higgs field is in the adjoint representation was performed in [19]. We found qualitative agreement with the results of ref. [19] for the model (6) in the rather large region of coupling constant space we have scanned. There are no surprises compared to the standard scenario for a four-dimensional gauge-Higgs system: for $\beta_{G}=0$ we have for small $\beta_{R}$ a first-order transition near $\beta_{H}=1 / 3$, separating the broken and unbroken phases. As $\beta_{R}$ increases the $\beta_{H}$ separating the two phases increases slightly, and the transition becomes progressively weaker first order, ending in a second-order transition at some finite $\beta_{H}, \beta_{R}$. This picture is preserved when we move away from $\beta_{G}=0$, and the value of $\beta_{R}$, which separates the first- and second order-transition regions, is lowered with increasing $\beta_{G}$. At the other boundary (i.e. $\beta_{G}=\infty$ ) the transition separating the broken and unbroken phase is always second order. When we move away from $\beta_{G}=\infty$ small values of $\beta_{R}$ will result in a first-order transition for $\beta_{H}$ close to $1 / 3$, while larger values of $\beta_{R}$ result in a second-order transition. In this way there exists a tricritical line 
$\tilde{\beta}_{R}\left(\beta_{G}\right), \tilde{\beta}_{H}\left(\beta_{G}\right)$ extending from the Gaussian fixed point $\beta_{G}=\infty, \beta_{R}=0, \beta_{H}=1 / 3$ and separating the first- and second-order transitions. We have not located precisely where it ends.

We now discuss the influence of the parity breaking term $S_{1}$. Let us define the lattice observable related to $\tilde{\mathcal{O}}_{1}^{\text {cont }}$ as follows:

$$
\mathcal{O}_{1} \equiv-\frac{<S_{1}>}{6 \mu<R^{2}>}
$$

The procedure has been to fix a value of $\mu \neq 0$ and scan the above phase diagram for the gauge-Higgs system and look for volume dependence of $\left\langle\mathcal{O}_{1}\right\rangle$. The first statement is that nowhere have we observed any volume dependence for $\left\langle\mathcal{O}_{1}\right\rangle$. The simulations were performed on $8^{3}, 12^{3}, 16^{3}$ and $24^{3}$ lattices. On the largest lattices it was possible to move quite close to the gaussian fixed point, but still there was no volume dependence. We conclude that we have seen no sign of spontaneous parity breaking.

We do observe, however, explicit parity breaking. No matter what value of $\beta_{R}, \beta_{H}$ and $\beta_{G} \neq 0$, a sufficiently large value of $\mu$ results in a phase transition (or for a large value of $\beta_{R}$ or $\beta_{H}$ at least a rapid cross-over) to a state with a large expectation value of $\mathcal{O}_{1}$. The corresponding phase diagram is shown in fig. 3 for $\beta_{G}=6$ and $\beta_{R}=0.001$. We have three phases denoted $S, S B$ and $P B . S$ denotes a symmetric phase where the expectation value of $R^{2}$ is small, the expectation value of the correlator

$$
\operatorname{Tr} V^{\dagger} U V \equiv \operatorname{Tr} V_{x}^{\dagger} U_{x, x+\mu} V_{x+\mu}
$$

between the phase of the Higgs field and the gauge field is small and finally the expectation value of $\mathcal{O}_{1}$ is small. The phase $S B$ denotes the phase of spontaneously broken symmetry. It is a continuation of the similar phase for the ordinary theory with $\mu=0$. In this phase $R^{2}$ is large, $\operatorname{Tr} V^{\dagger} U V$ is close to two, its maximum value, and $\mathcal{O}_{1}$ is small: there is only a small violation of parity, induced by the explicit presence of the term with $\mu$ in the action. The phase $P B$ is one where parity appears to be broken in the sense that the expectation value of $\mathcal{O}_{1}$ is large. Note that a positive expectation value of $\mathcal{O}_{1}$, due to the sign convention in (14), corresponds to a negative value for the action term $S_{1}$ given by (9). In this phase the expectation value of $R^{2}$ is large too, and $\mathcal{O}_{1}$ is approximately 0.6 of its maximal value. The expectation value of $\operatorname{Tr} V^{\dagger} U V$ is small, as is the expectation value of $\operatorname{Tr} U_{\square}$. The three phases meet at the "triple point" $T$. We have illustrated the behaviour in fig. 4 of the four observables mentioned. Deep inside the broken phase (large $\beta_{H}$ ) the transition is probably only a rapid cross-over and not a real phase transition. For the given value of $\beta_{G}$ and $\beta_{R}$ the transition between the symmetric phase $S$ and the 
parity broken phase $P B$ is a clear first-order transition, (it is sharp, independent of the volume and it is possible to produce a pronounced hysteresis loop ). Fig. 5 shows the effect of changing $\mu$ for a fixed $\beta_{H}$ which is chosen such that we start in the $S$ phase, afterwards move into the $S B$ phase and finally end in the $P B$ phase. Figs. $6 \mathrm{a}$ and $\mathrm{b}$ illustrate the effect of increasing $\beta_{R}$. The main effect of such an increase is that we have to go to somewhat larger $\mu$ to induce the transition to the $P B$ phase. The value of $\mu$ reported here is the value of the $\mu_{T}$ for the triple point where the three phases meet. The $\beta_{H}$ value for the triple point always remains smaller than the $\beta_{H}$ for $\mu=0$ and larger than $1 / 3$, which is the value for the Gaussian fixed point corresponding to $\mu=0$ and $\beta_{R}=0$. From fig. $6 \mathrm{~b}$ we have indications that the triple point moves closer to this gaussian critical point as $\beta_{R} \rightarrow 0$. Finally Fig. $6 \mathrm{c}$ illustrates the behaviour of $\mu_{T}$ versus $\beta_{R}$ and $\beta_{G}$. We observe first that $\mu_{T}$ tends to a finite value as $\beta_{G}$ tends to zero for constant $\beta_{R}$ (we will discuss the reason for considering this particular limit below). On the other hand $\mu_{T}$ increases with $\beta_{G}$, but if we want to follow the lines of constant physics as $\beta_{G}$ increases we must, according to the naive scaling relations, move to smaller $\beta_{R}$ (recall that $\beta_{R} \sim a$ and $\left.\beta_{G} \sim a^{-1}\right)$. Doing this we observe that $\mu_{T}$ on the lattice is almost constant, which indicates that $\mu_{1 c}$ approaches zero in a tentative continuum limit based on the naive scaling relation. But, as already mentioned, and as will be discussed in more detail below, the transition at $\mu_{T}$ is always a first-order transition and it makes no sense to apply the naive scaling relations.

Let us try to understand the nature of the new parity odd phase and examine in more detail the possibility of associating it with a continuum theory.

From fig. 4 the parity broken phase appears to be a lattice artifact. In particular, the plaquette variable $U_{\square ; \mu \nu}$ must rotate close to the equator of $S^{3}$ if we map $S U(2)$ in the standard way in the three-sphere, and the smallness of the plaquette action is not due to rapid fluctuations of the link variables, as in usual gauge theories, but is due to an unusual kind of alignment with the vector $H^{a}$. This is clear since $\mathcal{O}_{1}$ is approximately 0.6 of its maximal value. This alignment automatically prohibits the usual alignment between the phases of the gauge field and the Higgs field in the broken phase, and explains the observed small value of $\operatorname{Tr} V^{\dagger} U V$ in spite of the fact that the expectation $<R^{2}>$ of the Higgs field is large. The smallness of $\operatorname{Tr} U_{\square}$ itself, not only its expectation value, moves any expansion a long way from the continuum.

The possibility of a phase transition for large values of $\mu$ is not difficult to understand from a simple mean field argument. Adding an operator like $\mu \tilde{\mathcal{O}}_{1}^{\text {cont }}$, given by (2) (or $S_{1}$ given by (9) on the lattice) to the theory corresponds to adding, in a not too precise way, a tachyonic mass term to the theory, since it is quadratic in the field $\varphi$ and it will dynamically choose to adjust itself to a tachyonic coupling, 
rather than to a real mass term. (Recall that the expectation value of $S_{1}$ or $\mu \tilde{\mathcal{O}}_{1}^{\text {cont }}$ was negative.) Of course it has to compete with the real mass terms present in the theory, but whenever $\mu$ is sufficiently large the tachyonic mode will have the chance to dominate, provided a suitable alignment of the gauge fields can be found. By just using the mean field values for the action terms associated with $\beta_{G}, \beta_{H}$ and $\beta_{R}$ it is possible to predict the value of $\mu$ for which the phase transition to a "broken parity" phase should occur as a function of $\beta_{G}, \beta_{H}, \beta_{R}$. It agrees reasonably with the observed value. Under the assumption that $\operatorname{Tr} V^{\dagger} U V=0$ and $U_{\square}=0$, which is approximately satisfied deep in the $P B$ phase, we can find a constant field configuration which seems to agree with the observations. By a gauge fixing to the unitary gauge we assume $V_{x}=1$ and $\operatorname{Tr} V^{\dagger} U V=0$ reduces to $\operatorname{Tr} U_{\mu}=0$ for all links. This in combination with $\operatorname{Tr} U_{\square}=0$ tell us that

$$
U_{\square}=i \sigma^{a} u_{\square}^{a}, \quad U_{\mu}=i \sigma^{a} u_{\mu}^{a} .
$$

Since $U_{\square}$ is a product of four $U_{\mu}$ 's it is simple algebra to show that (16) completely fixes the vectors $u_{\mu}$ corresponding to links in the 1,2 and 3 directions to satisfy

$$
u_{\mu}^{a} u_{\nu}^{a}= \pm 1 / \sqrt{2}, \quad \mu \neq \nu=1,2,3
$$

This in turn implies that

$$
\frac{1}{6} \varepsilon_{\mu \nu \lambda} H_{\mu}^{a} G_{\nu \lambda}^{a} / R^{2}= \pm \frac{1}{6} \varepsilon_{\mu \nu \lambda} \varepsilon^{a b c} u_{\mu}^{a} u_{\nu}^{b} u_{\lambda}^{c}= \pm \sqrt{\sqrt{2}-1}= \pm 0.645
$$

and the appropriate choice of sign will give a negative value of $S_{1}$ which is close to the observed value deep in the $P B$ phase.

Although these mean field arguments are valid deep inside the $P B$-phase, they are likely to fail close to the transition between the symmetry phase $S$ and the phase $S B$. For $\mu=0$ and suitable values of $\beta_{G}, \beta_{H}$ and $\beta_{R}$ we have a secondorder transition and this transition seems to persist for small values of $\mu$. We have therefore observed carefully whether there is any sign of enhancement of parity breaking for a fixed small $\mu$, when we move from the symmetric to the broken Higgs phase (we assume the value of $\mu$ so small that we do not have the phenomenon of tachyonic transition described above). In fig. 7 we have shown such a curve for $\mathcal{O}_{1}$ as a function of $\beta_{H}$, and we have observed an enhancement of the parity breaking transition precisely at the phase transition. Unfortunately there was still no volume dependence associated with the observed expectation value of $\mathcal{O}_{1}$, and further the response was linear with $\mu$. Again we could not associate it with any sign of spontaneous parity breaking, and one possible interpretation of the enhancement is simply that it represents a cross-over from one kind of field configuration, which has only a weak response to the explicit parity breaking $S_{1}$ present in the action, 
namely the configurations in the unbroken phase, to another, completely different, kind of configuration, in the broken phase, which has an equally weak dependence. In between these two well-defined phases we might get interpolating configurations which by "accident" trigger a somewhat larger value of $\mathcal{O}_{1}$.

One could ask whether it is possible to use the explicit parity breaking observed to define a continuum limit. The first step in that direction is to locate second-order transitions. Two locations are possible: in the first case, the transition is reached from the symmetric phase of the gauge-Higgs system by increasing the "chemical" potential $\mu$, and in the second case it is reached from the broken phase of the gauge-Higgs system by the same procedure. A priori the first situation seems much more interesting, since the symmetric phase is non-perturbative and is infra-red singular from a perturbative point of view. However, whenever we approached the line of transitions from the unbroken phase we observed clearly the phenomenon of hysteresis, indicating a first-order transition. It seems unlikely that this part of the critical line can be used to define any continuum theory. Although it was more difficult to decide whether the transition in the broken phase was a first- or second-order transition, it is not very appealing to think of this critical line as one where continuum physics can be defined. The "perturbative vacuum" where $\mu=0$ is certainly well defined and stable in this phase. Well into the parity broken phase we have a situation which differs profoundly from any known continuum assignment of gauge fields to link variables, and it is hard to imagine the borderline between these two regimes as being interesting.

One point is singled out, namely the triple point $T$ where the three phases meet. For $\mu=0$ we expect the transition between the symmetric and broken Higgs phases to be second order if $\beta_{G}$ is sufficiently large and $\beta_{R}$ not too small. This feature seems to extend to $\mu>0$, and it might extend all the way to the triple point $T$ of fig. 3 , in which case one could imagine using this second-order point to define a continuum limit. However, for all the coupling constants we have checked the situation has been as follows. Even if the transition between the phases $S B$ and $S$ starts out as a second order transition for $\mu=0$, and continues to be second-order for $\mu$ small, it always changed to a first-order transition before we reached the triple point. We illustrate this situation in fig.8. For $\beta_{G}=5.0$ and $\beta_{R}=0.01$ we plot $1 / 2 \operatorname{Tr} V^{\dagger} U V$ as a function of $\beta_{H}$ for two values of $\mu: \mu=0$. and $\mu=0.16$. The second-order transition at $\mu=0$ has changed into a clear first-order one for $\mu=0.16$. Finally we plot $1 / 2 \operatorname{Tr} V^{\dagger} U V$ and $<\mathcal{O}_{1}>$ versus $\mu$ for a value of $\beta_{H}$ near the triple point. Again we see a first-order transition.

We conclude that we have not been able to find a second-order phase transition point between $S$ and $P B$. 
For larger $\beta_{H}$ we will have a transition between $S B$ and $P B$. When we move away from the triple point and up in $\beta_{H}$ this transition is first order, but eventually it seems to change into a cross-over. Also in this region we found no obvious secondorder transition which could serve as a definition for a continuum limit.

\section{The phase diagram with $\tilde{\mathcal{O}}_{3}^{\text {cont }}$ in the action}

As mentioned in the introduction we expect the term $\tilde{\mathcal{O}}_{3}^{\text {cont }}$ to differ significantly from $\tilde{\mathcal{O}}_{1}^{\text {cont }}$ in the symmetric phase $S$. We also expect it to be a better approximation to a Chern-Simons like term. For this reason we have repeated the analysis of the phase structure with this term added instead of $\tilde{\mathcal{O}}_{1}^{\text {cont }}$. As the lattice version we have taken

$$
S_{3}=4 \mu \sum_{x} \varepsilon_{\mu \nu \lambda} H_{\mu}^{a}(x) G_{\nu \lambda}^{a}(x) / R^{2}(x)
$$

The continuum coupling constant $\mu_{3 c}$ which multiplies $\tilde{\mathcal{O}}_{1}^{\text {cont }}$ is dimensionless and if we apply naive scaling, we have $\mu \sim \mu_{3 c}$ without any lattice scale $a$ entering.

In fig. 9 we have shown a phase diagram similar to the one shown in fig. 3 for two values of $\beta_{R}$. The qualitative features are the same as in fig.3 except for the expectation value of $\left\langle R^{2}\right\rangle$, which seems to be continuous when we cross from the symmetric phase $S$, to the one of spontaneous parity breaking $P B .\left\langle R^{2}\right\rangle$ stays small everywhere in $P B$.

In fig.10 we illustrate the behaviour of our observables as a function of $\mu$. We have chosen $\beta_{G}=6.0, \beta_{R}=0.001$ and two values of $\beta_{H}$ corresponding to a location below and just above the triple point.

Again we see no sign of spontaneous parity breaking for small $\mu$, but a transition for large values of $\mu$ to a parity broken phase $P B$. The transition from $S$ to $P B$ is always first order, even at the triple point $T$, and the mean field arguments still apply, since they do not use any properties of $\left\langle R^{2}\right\rangle$.

The nature of the transition from $S B$ to $P B$ depends on the value of $\beta_{R}$. For $\beta_{R}=0.001$ it is always first-order even for large values of $\beta_{H}$, but for $\beta_{R}=0.1$ it starts as a first order transition for $\beta_{H}$ just above the value corresponding to the triple point. For $\beta_{H}=0.50$ the transition is weakly first order, while for $\beta_{H}>0.60$ it has changed into a cross-over.

As for the $\tilde{\mathcal{O}}_{1}^{\text {cont }}$ term we have to conclude that there seems to be no secondorder phase transition which can be used for defining a continuum limit of the parity broken phase. 


\section{A "Chern-Simons" condensate}

Although the parity broken phase $P B$ is a lattice artifact, it is still of some interest to check whether it possesses some of the properties which were conjectured for the hypothetical continuum version of the parity broken phase. The most important property was the existence of a Chern-Simons condensate, which during the phase transition from the parity broken phase at time $t_{1}$ to the "present" phase of spontaneously broken gauge symmetry at time $t_{2}$ would develop a large value of

$$
N_{c s}\left(t_{2}\right)-N_{c s}\left(t_{1}\right)=\frac{1}{16 \pi^{2}} \int_{t_{1}}^{t_{2}} d t \int d^{3} x \operatorname{Tr} \tilde{F}_{\mu \nu} F_{\mu \nu}
$$

where the Chern-Simons number $N_{c s}(t)$ is defined by

$$
N_{c s}(t)=\int d^{3} x n_{c s}(x, t)
$$

While $N_{c s}(t)$ itself is not invariant under topological non-trivial gauge transformation, the difference given in (20) is invariant. Consequently, it makes sense to ask for this value in the case of a given phase transition (first order, second order etc.) from the $P B$-phase to the $S B$-phase. As explained in the introduction we will mainly be interested in the situation where the electroweak transition is of first orderf. A firstorder transition is well approximated by a simple change in the coupling constants [20]. An approximation to the first-order electroweak transition can then be implemented in the following way. Our starting point is the parity broken phase, which we have reached from the symmetric phase (corresponding to the high temperatures before the electroweak transition). We then change the coupling constants such that they correspond to the broken phase, and relax the gauge field configuration such that it has a possibility to move to a classical vacuum configuration in the broken phase. During this motion we measure the lattice version of the rhs of (20) (for a detailed discussion of this program see [21, 22]).

One has a large choice of possible relaxation equations. One possible choice would be the classical equations of motion with a damping term (to take away energy from the hot configuration). Another choice, which we have adopted here, is to use the simplest relaxation equation which will bring us to a classical vacuum in the broken phase:

$$
\frac{\partial \varphi(x, \tau)}{\partial \tau}=-\frac{\delta S}{\delta \varphi(x, \tau)}, \quad \frac{\partial A(x, \tau)}{\partial \tau}=-\frac{\delta S}{\delta A(x, \tau)} .
$$

\footnotetext{
${ }^{4}$ In order to avoid any misunderstanding, let us stress that the question of a first- or secondorder electroweak transition cannot be addressed in the present setup. Since we are working in the approximation where dimensional reduction is assumed, we cannot ask for any dynamics associated with temperature. The discussion in the former sections concerning first- and secondorder transitions only refers to the possibility of defining a three-dimensional continuum theory from the lattice theory.
} 
where $S$ is the action for the gauge-Higgs system and $(A, \varphi)$ symbolizes the gaugeand Higgs fields.

The "time" $\tau$ entering in (22) is a fictitious relaxation time, but (20) is independent of the choice of time-parameter since it depends only on the initial and final field configurations. Of course the final configuration depends on the specific choice of dynamics dictated by (22), but since the final configuration is a trivial vacuum configuration, where only the winding number is of importance, we do not expect a crucial dependence on the choice of dynamics during the "rolling down" of the Higgs field. At least, the result of relaxation is invariant to small perturbations of the choice of relaxation equations since the classical vacua are separated points of attraction of any relaxation equation.

The measurement of the "Chern-Simons condensate", i.e. of (20) according to the procedure described above, is shown in fig.11, both for the condensate generated from a local parity odd term $\tilde{\mathcal{O}}_{1}^{\text {cont }}$ and the non-local parity odd term $\tilde{\mathcal{O}}_{3}^{\text {cont }}$. We see a clear condensate, where (20) grows proportional to the volume (the density goes to a constant value with volume), although we have to go to rather large volumes to see this clearly in the case of $\tilde{\mathcal{O}}_{3}^{\text {cont }}$.

Fig.12 shows the dependence of the "Chern-Simons condensate" on $\mu$ for the lattice version of local action $\tilde{\mathcal{O}}_{1}^{\text {cont }}$ and for constant values of $\beta_{R}, \beta_{G}$ and $\beta_{H}$. In the same figure we plot for comparison the value of $\left\langle\mathcal{O}_{1}\right\rangle$ and we see that the condensate is only formed after the transition to the parity broken phase.

\section{Discussion}

We have seen no sign of spontaneous breaking of parity. One could try to discard these lattice results by saying that the lattices are too small, and that we are far from continuum physics. However, from the simulations of pure $S U(2)$ gauge theories in three dimensions it is known 23] that the scaling region is reached already at $\beta_{G}=5$ and that the correlation length at this value is quite small. For the pure gauge theory we can therefore approach continuum physics on quite small lattices. Of course the gauge-Higgs system is different and the question of spontaneous parity breaking a different one. The hope of such a phenomenon being present is nevertheless born from the infra-red singularities present in the gauge-Higgs system in the symmetric phase, and there is no obvious reason why the scales involved should be vastly different from the ones which are believed to control the infra-red singularities of the pure gauge system.

The most natural interpretation of the results is as follows. The infra-red sin-

gularities in three-dimensional non-Abelian gauge theories (and the magnetic sector 
of high temperature four-dimensional non-Abelian gauge theories) can be cured by a dynamical generated mass scale. We believe such a mass scale is generated for the string tension[23] and the glueball mass [24, 25] in the symmetric phase. This mass scale could then counteract the potential instability coming from a ChernSimons term. We can write down the following toy model quadratic effective action in Euclidean space:

$$
\int d^{3} p \frac{1}{2} A_{k}(p)\left[\left(p^{2} \delta_{i k}-p_{i} p_{k}\right)+m^{2} \delta_{i k}+i \mu \varepsilon^{i j k} p_{j}\right] A_{i}(p)
$$

This action is of course not meant to be taken seriously, since no reference is given to the non-Abelian nature of the interaction. It is meant to serve as an order of magnitude estimate of the effects to be discussed. If $m=0$ the modes with momenta $|p|<\mu$ are tachyonic and one would expect a condensation of the modes $A_{i}(p)$ which are only stabilized if we include $A^{4}$ terms in the effective action. If $m>0$ it requires a finite value of $\mu$ to trigger a condensation to a parity broken phase. The above reported results are in qualitative agreement with the existence of such a mass scale which would prevent any spontaneous parity breaking. In fact we can, somewhat courageously, use the toy model (23) to estimate the non-perturbative mass $\mathrm{m}^{2}$ which enters in the action, since we have determined the critical $\mu$-value where the tachyonic mode begins to dominate. The most obvious comparison is made between the lattice version of $\tilde{\mathcal{O}}_{3}^{\text {cont }}$ and (23), since the non-local lattice action is closest to the Chern-Simons like term in (23). After a little algebra we find that the naive relation between the $\mu$ which appears in (23) and $\mu_{3}$ multiplying the lattice action is given by

$$
\mu a=\frac{8 \mu_{3}}{\beta_{G}}
$$

where $a$ denotes the lattice spacing. We conclude from the observed critical values of $\mu_{3}$ that

$$
m \equiv \mu=\mathcal{O}(1) / a
$$

The inverse mass seems to be of the order of the lattice spacing. At first one would be tempted to dismiss this result and simply say that $\mu$ is of the order of the typical lattice excitations, which have nothing to do with continuum physics. This might be true, but the remarkable fact is that this very large "non-perturbative" mass scale is precisely what we observe in three-dimensional lattice gauge theories, whether one likes it or not. It is not our task here to discuss whether the non-perturbative mass-scales observed in 224, 25] are reasonable or not, we can only conclude that our results are fully consistent with the non-perturbative results in [23, 24, 25].

The large value of the inverse magnetic scale $m_{\text {magn }} \sim\left(2 \mu_{3}\right) g^{2} T \sim 3 g^{2} T$ introduces large uncertainties in many quantities connected with the electroweak 
baryogenesis scenario. First, any analytical scheme for the calculation of the finite temperature effective potential fails for $M_{W}(\phi)<m_{\text {magn }}$ due to the infra-red divergences. This means that we do not know the shape of the effective potential at $\phi<6 g T$, so that it is very difficult to analyse the dynamics of the phase transition, for which the behaviour of the potential at small $\phi$ is essential. Moreover, as has been pointed out in [12], the vacuum expectation value for the Higgs field should be sufficiently large in order to suppress B-violation right after the phase transition, namely $\phi>3 g T$. Due to the fact that $\mathrm{SU}(2)$ gauge coupling is not so small, we cannot trust any analytic treatment of the scalar effective potential even for so large $\phi$. Second, the calculation of the rate of the fermionic number non-conservation at temperatures below the phase transition temperatures relies heavily on the smallness of the non-perturbative magnetic effects. As we see, our analysis favours a large magnetic screening mass, so that there are large uncertainties in the calculation of the rate too. In other words, two basic ingredients which can be used for imposing an upper limit on the Higgs mass from cosmology are not quite established at the moment.Certainly, much more numerical work should be done in this direction.

We can now ask whether there are continuum theories which have any similarities to the explicit parity broken phase we have seen on the lattice. The theory of cold neutral fermionic matter in $(V-A)$ gauge theories considered in [3] has many similarities. In the presence of a chemical potential $\mu$ for fermions the effective Hamiltonian of the bosonic fields will essentially be given by the gauge-Higgs system we have considered in this article plus a Chern-Simons term where the coupling constant is proportional to $\mu$. The Lagrangian is given by

$$
-\mathcal{L}=\frac{1}{2 g^{2}} \operatorname{Tr} F_{\mu \nu}^{2}+\left|D_{\mu} \varphi\right|^{2}+\lambda\left(|\varphi|^{2}-\varphi_{0}^{2}\right)^{2}+\sum_{i=1}^{f} \bar{\psi}_{L}^{(i)} i \sigma_{\mu} D_{\mu} \psi_{L}^{(i)}
$$

The gauge group is $S U(2), \varphi$ is a scalar doublet and $f$ denotes the number of lefthanded fermions. The authors in [3] studied a situation where there was a fermionic density, neutral with respect to all gauge charges. Integrating out the fermions led to the already mentioned Chern-Simons term and they have shown that in the case where $\lambda<<g^{2}$ the normal vacuum

$$
\varphi=\varphi_{0}, \quad A_{i}=0
$$

is replaced by a more complicated state where

$$
\varphi=0
$$

and where a gauge field condensate with a Chern-Simons number different from zero is created. Our lattice system seems to realize this situation when coupled to the 
lattice version of $\tilde{O}_{3}^{\text {cont }}$. The parity broken phase $P B$ has the same expectation value of $|\varphi|^{2}$ as one finds in the symmetric phase, and a Chern-Simons-like condensate is present. Ref. [3] only discusses one of an infinite number of contributions to the effective Hamiltonian which arises when one integrates out the fermions from the underlying theory. In the same way we have here only considered the lattice version of one of these terms in the effective action and the final result was a condensate which was a lattice artifact, but which nevertheless has the essential features of the condensate suggested in [3].

Still another model which exhibits explicit parity breaking is the three-dimensional Abelian gauge-Higgs system with a Chern-Simons term[26]:

$$
S=-\int d^{3} x\left|D_{\mu} \phi\right|^{2}+V(\phi)+\frac{1}{4} \varepsilon^{\mu \nu \lambda} A_{\mu} F_{\nu, \lambda}
$$

where $D_{\mu}=\partial_{\mu}-i e A_{\mu}$ and $V(\phi)$ is a Higgs potential. In the absence of the Maxwell term $\frac{1}{4} F_{\mu \nu}^{2}$ the gauge field is non-dynamical and if one expands around the false vacuum $\phi=0$ there are just two scalar modes. However, if one expands around the true vacuum $\phi=\phi_{0}$ the effective vector action is

$$
S=-\int \frac{m}{2} A_{\mu}^{2}+\frac{1}{4} \varepsilon^{\mu \nu \lambda} A_{\mu} F_{\mu \nu}
$$

where $m=e^{2} \phi_{0}^{2}$. This first-order system has an alternative formulation as a conventional second-order system describing a topological massive spin 1 particle, with the $A_{\mu}$ essentially the dual of the Maxwell field strength. Thus the absorbed Higgs scalar has been converted into an odd parity helicity 1 excitation.

Our model is different from (29) in several aspects. It is non-Abelian, it has a "Maxwell" term and it is living in Euclidean space-time. Nevertheless, it might for extreme values of the coupling constants have a parity odd phase where the excitations are of a similar nature. In order to investigate this possibility we have performed lattice simulations for very small values of $\beta_{G}$ such that the independent dynamics of the gauge field is decoupled. However, the conclusion was the same as for the more conventional values of $\beta_{G}$. Nowhere we could find a candidate for a second-order transition between the $S B$-phase and the $P B$ phase. The non-Abelian nature of the gauge group presumably makes the model radically different from (29).

Acknowledgement. We thank V. Rubakov and S.Khlebnikov for discussions. Computer time on an Amdahl VP1100 has been financed by the Danish Research Council. 


\section{References}

[1] G. 't Hooft, Phys. Rev. Lett. 37 (1976) 8; Phys. Rev. D14 (1976) 3432.

[2] V. A. Kuzmin, V. A. Rubakov and M. E. Shaposhnikov, Phys. Lett. 155B (1985) 36.

[3] V. A. Rubakov and A. N. Tavkhelidze, Phys. Lett. 165B (1985) 109; V.A. Rubakov, Prog. Theor. Phys. 75 (1986) 366; V.A. Matveev, V.A. Rubakov, A.N. Tavkhelidze and V.F. Tokarev, Nucl. Phys. B282 (1987) 700.

[4] V.A. Rubakov, JETP Lett. 41 (1985) 266; Nucl.Phys. B256 (1985) 434.

[5] A. Ringwald, Nucl. Phys. B330 (1990) 1.

[6] O. Espinosa, Nucl. Phys. B334 (1990) 310.

[7] T. Appelquist, M. Bowick, D. Karabali and L.C.R. Wijewardhana, Phys.Rev. D33 (1986) 1774.

[8] T. Appelquist and D. Nash, Phys.Rev.Lett. 64 (1990) 721.

[9] G. Semenoff and L.C.R. Wijewardhana, Phys.Rev.Lett. 63 (1989) 2633.

[10] S. Yu. Khlebnikov, Pisma ZhETF 51 (1990) 69.

[11] S. Y. Khlebnikov and M.E. Shaposhnikov, Phys.Lett. 254B (1991) 148.

[12] M.E. Shaposhnikov, Nucl.Phys B287 (1987)757; Nucl.Phys. B299 (1988) 797.

[13] M.E. Shaposhnikov, Phys. Lett. B277 (1992) 324.

[14] M.E.Carrington, "The effective potential at finite temperature in the standard model",TPI-MINN-91/48-T; M.Dine,R.G.Leigh P.Huet,A.Linde and D.Linde, "Comments on the electroweak phase transition", SLAC-PUB-5740.

[15] B.Bunk, E.M.Ilgenfritz,J.Kripfganz, and A.Schiller, "Lattice studies at zero and finite temperature in the $\mathrm{SU}(2)$ Higgs model at small couplings", preprint BI-TP 92/12 (1992).

[16] A.D.Linde, Phys.Lett 96B (1980) 289.

[17] D.Gross, R.Pisarski and L.Yaffe, Rev. Mod. Phys. 53 (1981) 43.

[18] A.Redlich and L.C.R. Wijewardhana, Phys.Rev.Lett. 54 (1985)970;

A.Niemi and G.Semenoff, Phys.Rev.Lett. 54(1985)2166;

A.Tsokos, Phys.Lett. 157B (1985)423. 
[19] S. Nadkarni, Nucl.Phys B334 (1990) 559; K. Farakos, G. Koutsoumbas, Z.Phys. C43 (1989) 301.

[20] A.D. Linde, Nucl.Phys. B216 (1983) 421.

[21] J. Ambjørn, M. Laursen and M.E. Shaposhnikov, Phys.Lett 197B (1987) 49; Nucl.Phys. B316 (1989)483.

[22] J. Ambjørn, T. Askgaard, H. Porter and M.E. Shaposhnikov, Phys.Lett 244B (1990) 479; Nucl. Phys. B353 (1991) 346.

[23] J. Ambjørn, P. Olesen and C. Peterson, Nucl.Phys. B240 (1984) 553; Phys.Lett 142B (1984) 410;

[24] A. Irback and C. Peterson, Phys.Lett.174B (1986) 99.

[25] K. Farakos, G. Koutsoumbas and S. Sarantakos, Phys.Lett. 189B (1987) 173.

[26] S. Deser and Z. Yang, Mod.Phys.Lett A4 (1989) 2123. 


\section{Figure caption}

Fig.1 Triangle diagram which results in the effective term $\tilde{\mathcal{O}}_{1}^{\text {cont }}$ given by (2) in the high temperature limit

Fig.2 (a): $<\mathcal{O}_{1}>$ as a function of the coupling strength $\mu$ in the case of spontaneous parity breaking. For a fixed $\mu$ we expect $\left\langle\mathcal{O}_{1}\right\rangle$ to grow with the volume of the system. (b): The same functions as in (a), but with no parity breaking.

Fig.3 The phase diagram for $\beta_{G}=6.0$ and $\beta_{R}=0.001$ and the action corresponding to $\mathcal{O}_{1}$. The used lattice size is $8^{3}$. $S$ denotes the symmetric phase, $S B$ the spontaneously broken phase, while $P B$ means the parity broken phase.

Fig.4 The behaviour of four observables as a function of $\mu$ for $\beta_{G}=6.0$ and $\beta_{R}=$ 0.001 for three different values of $\beta_{H}$, which for $\mu=0$ corresponds to the symmetric phase $\left(\beta_{H}=0.35\right.$, shown with black dots), just inside the spontaneously broken phase $\left(\beta_{H}=0.36\right.$, shown with triangles) and deep inside the broken phase $\left(\beta_{H}=0.42\right.$, shown with open circles).

Fig. $5<\mathcal{O}_{1}>$ (black dots) and $\frac{1}{2}<\operatorname{Tr} V^{\dagger} U V>$ (triangles) for $\beta_{R}=0.001, \beta_{G}=6.0$ and $\beta_{H}=0.353$. The value is chosen in such a way that we start in phase $S$ for $\mu=0$. With increasing $\mu$ we move into phase $S B$ as seen from the jump in $\frac{1}{2}<\operatorname{Tr} V^{\dagger} U V>$, and finally we move into phase $P B$, where $<\mathcal{O}_{1}>$ jumps while $\frac{1}{2}<\operatorname{Tr} V^{\dagger} U V>$ decreases.

Fig.6 (a): The phase transition to $P B$ for various values of $\beta_{R}$. For a given value of $\beta_{R}$ the open circles correspond to a transition from $S B$ to $P B$ while the black dots correspond to a transition from $S$ to $P B$. (b): The location of the "triple point" $T$ of fig. 3 in the $\mu, \beta_{H}$ plane as a function of $\beta_{R}$. The four points correspond to $\beta_{R}=0.001,0.005,0.01$ and 0.05 , both $\beta_{H}$ and $\mu$ increasing with $\beta_{R}$. (c): The dependence of $\mu_{T}$ on $\beta_{R}$ and $\beta_{G}$.

Fig.7 The expectation value $<\mathcal{O}_{1}>$ (open circles) for a fixed value of $\mu$ as a function of $\beta_{H}$. We are close to $\mu=0.0$ and the peak appears at the transition between the phase $S$ and $S B$, as is seen from the expectation value of $\frac{1}{2} \operatorname{Tr} V^{\dagger} U V$ (triangles ).

Fig.8 The change in the order of the phase transition from (1): $S$ to $P B$ as a function of $\beta_{H}$ (fig.8a and fig.8b), and from (2): $S B$ to $P B$ as a function of $\mu$ (fig.8c and fig.8d).

Fig.9 (a): the phase diagram for $\beta_{G}=6.0$ and $\beta_{R}=0.001$ and the action corresponding to $\mathcal{O}_{2}$. (b): the phase diagram for $\beta_{G}=6.0$ and $\beta_{R}=0.01$. The used lattice size is $8^{3}$. $S$ denotes the symmetric phase, $S B$ the spontaneously broken phase, while $P B$ means the parity broken phase.

Fig.10 Fig.10a:The expectation value of $R$ as a function of $\mu$ for two different values of $\beta_{H}(0.1$ (circles) and $0.4($ dots $))$ and $\beta_{R}=0.001, \beta_{G}=6.0$. The $\beta_{H}=0.1$ data show a transition between $S$ and $P B$, while the $\beta_{H}=0.4$ data show a transition between $S B$ and $P B$. Fig.10b and Fig.10c show $\left\langle\mathcal{O}_{3}>\right.$ and $\frac{1}{2}<\operatorname{Tr} V^{\dagger} U V>$ for the same values of coupling constants. 
Fig.11 The measurements of Chern-Simons condensates density as a function of lattice size for the local and non-local sources (circles and dots).

Fig.12 The expectation value of the Chern-Simons condensate versus $\mu$ for $\beta_{R}=$ $0.001, \beta_{H}=0.4$ and $\beta_{G}=9.0$ (circles) and (for comparison) the expectation value of $\left\langle\mathcal{O}_{1}\right\rangle$ (dots).

Fig.13 Measurement of the time-integral of $\tilde{F} F$ (eq. (20)) during cooling from the parity broken phase to the ordinary phase of spontaneously broken gauge symmetry. (a) For the local action, ( b) For the non-local action. 